\title{
Observing Hot Stars in all Four Stokes Parameters
}

\author{
Thomas Eversberg ${ }^{1}$, Anthony F.J. Moffat ${ }^{1}$, Michael Debruyne ${ }^{2}$, John B. \\ Rice $^{3}$, Nikolai Piskunov ${ }^{2,4}$, Pierre Bastien ${ }^{1}$, William H. Wehlau ${ }^{2}$, and \\ Olivier Chesneau ${ }^{5}$ \\ 1 Université de Montréal, Canada \\ 2 University of Western Ontario, Canada \\ 3 Brandon University, Canada \\ 4 Uppsala University, Sweden \\ ${ }^{5}$ Observatoire de la Côte d'Azur, France
}

\begin{abstract}
We introduce a new polarimeter unit which, mounted at the Cassegrain focus of any telescope and fiber-connected to a fixed CCD spectrograph, is able to measure all Stokes parameters $I, Q, U$ and $V$ photon-noise limited across spectral lines of bright stellar targets and other point sources in a quasi-simultaneous manner. We briefly outline the technical design of the polarimeter unit and the linear algebraic Mueller calculus for obtaining polarization parameters of any point source. In addition, practical limitations of the optical elements are discussed. We present first results obtained with our spectropolarimeter for three prototype hot-star.
\end{abstract}

\section{The polarimeter unit}

The newly built William-Wehlau-Spectropolarimeter (WWS) ${ }^{1}$, a collaboration between the University of Western Ontario, Brandon University and Université de Montréal, consists of two rotatable quarter-wave plates (QWP, as retarder) and a Wollaston prism (as beam-splitter and double-beam polarizer), leading light from stellar point sources via a double fiber-feed into a fixed CCD slit spectrograph.

Figure 1 shows the basic design of the polarimeter unit.

The WWS is designed to be mounted at the $\approx \mathrm{f} / 8$ Cassegrain focus of medium or large size telescopes. The principle light track:

Input aperture (pinhole) $\rightarrow$ Collimator $\rightarrow$ Glan-Taylor prism (removeable, produces $100 \%$ linearly polarized light) $\rightarrow$ Two rotatable QWP's (achromatic, $\mathrm{PC}$ controlled) $\rightarrow$ Wollaston prism (beamsplitter) $\rightarrow$ Camera $\rightarrow$ Twin optical fibers $\rightarrow$ Spectrograph slit $\rightarrow$ CCD.

${ }^{1}$ The original PI for this instrument, William H. Wehlau, unfortunately passed away in February 1995. We wish to express our deepest respect for his high level of competence and leadership during the planning and early construction stages of the spectropolarimeter that now carries his name. 


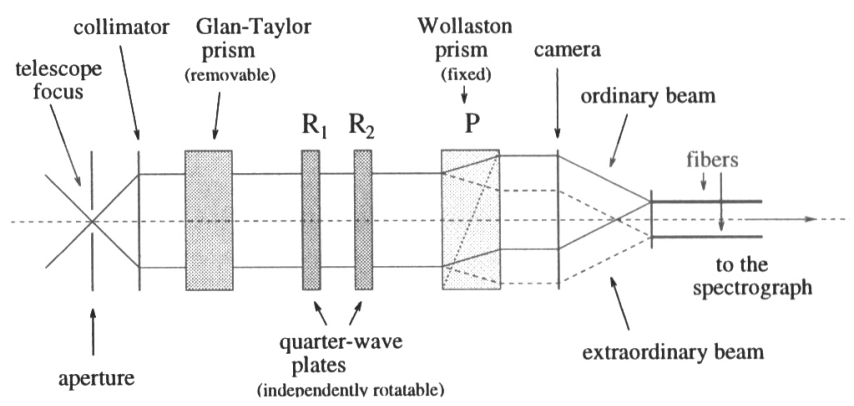

Fig. 1. Simple sketch of the Williarn-Wehlau-Spectropolarimeter.

The independent rotation of the QWP's relative to the Wollaston prism determines the polarization components that are being measured. The spectra of the light from each fiber are imaged so that they are parallel and adjacent, with sufficient space between them on the CCD detector of the spectrograph. The two components can be combined over an observing sequence to obtain all four Stokes' parameters, that fully define the polarization of the light. When the light is dispersed by the spectrograph, the polarization of the starlight may be calculated as the wavelength-dependent quantities $I(\lambda), Q(\lambda), U(\lambda)$ and $V(\lambda)$ via the Mueller calculus. For a full description of the instrument, the Mueller calculus and the data reduction, we refer to Eversberg (1998a, PASP, in press). We refer to Serkowski (1974, in: Methods of Exp. Phys, Vol. 12, Chap. 8) and the references therein as a basic source for the description of the Mueller calculus and different optical devices in this nomenclatura.

\section{Technical problems and first results}

We report some basic problems with our instrument:

Minor: SLIGHT DEPENDENCE of RETARDATION $\tau$ ON QWP POSITION ANGLE $\phi$. The QWP frames (worm-gears) as well as some QWP crystal layers are tilted with respect to the instrumental optical axis. This introduces a wobbling effect which can be corrected with a parametric fit for $\tau(\phi)$. This is clearly a manufacturing problem and airspaced QWP's are recommended to remedy this problem.

Major: Stochastic broadband polarization variability $Q, U$ and $V$ due to small variations in overall illumination of the two fibers, whose spatial surface sensitivities are never perfectly uniform (see also Donati et al. 1997, MNRAS, 291, 658, who report the same problem). As a consequence we can neither measure absolute values for $Q, U$ and $V$ nor the polarization angle at the sky. Smoothness of fiber apertures can possibly remedy this behavior. 

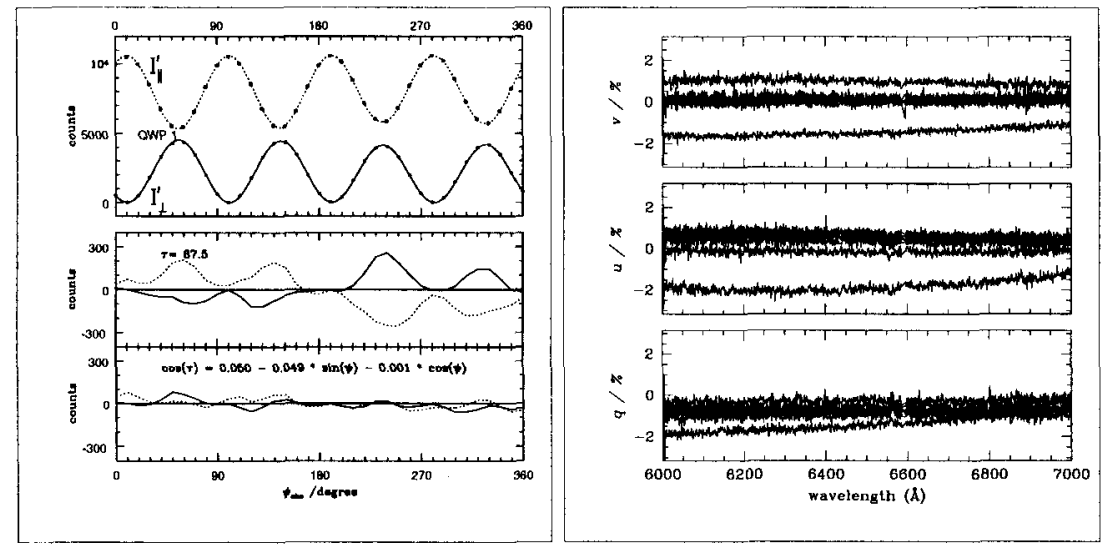

Fig. 2. Left: Top panel: Position angle dependence of the retardation $\tau(\phi)$ with $100 \%$ linear input polarization (Glan-Taylor prism). Center panel: $\chi^{2}$ fit with fixed $\tau=87.5^{\circ}$. Bottom panel: Parametric fit with $\tau(\phi)$ as indicated. Right: Seven consecutive sequences of $\gamma$ Cas during one night at Mont Mégantic Observatory.

The position angle dependence of the retardation $\tau(\phi)$ and a parametric fit is shown in Fig. 2 (left). An example for consecutive sequences of Stokes $Q, U$ and $V$ is also given in Fig.2 (right).

As a consequence, we cannot estimate the exact continuum polarization. For this reason we simply subtracted a fit to the original individual

Stokes $Q, U$ and $V$ spectra in each sequence and thus neglected broadband polarization, when combining sequences to get rectified mean $q(\lambda), u(\lambda)$ and $v(\lambda)$ spectra.

We confirm previous results by Poeckert \& Marlborough (1977, ApJ, 218, 220) for the Be star $\gamma$ Cas, find a feature in Stokes $v$ consistent with the measured magnetic field of $B_{e}=28000 \mathrm{G}$, in the Ap star $53 \mathrm{Cam}$ (Borra \& Landstreet 1977, ApJ, 212,141 ) and report newly observed line effects in $q$ and $u$ in the WR+O star $\gamma^{2}$ Vel (Eversberg et al. 1998b, submitted to ApJ).

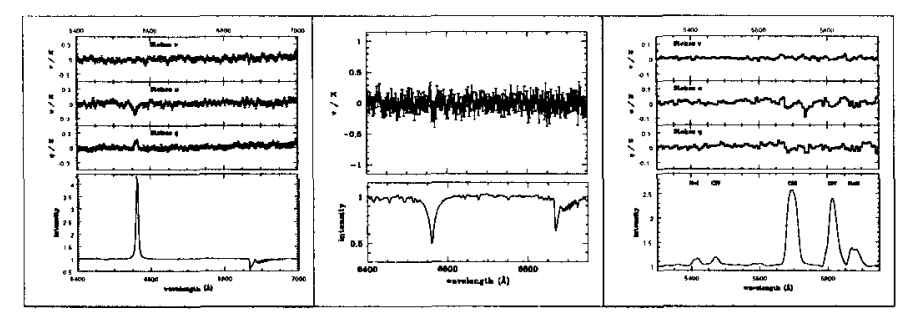

Fig. 3. Left: Stokes $q, u$ and $v$ for the Be star $\gamma$ Cas. Center: Stokes $v$ for the Ap star 53 Cam. Right: Stokes $q, u$ and $v$ for the WR $+\mathrm{O}$ binary $\gamma^{2}$ Vel. Nightly average intensity spectra are at the bottom of each panel. 


\section{Discussion}

H. Henrichs: How does your instrument compare with the MUSICOS polarimeter in terms of efficiency and throughput?

T. Eversberg: You have to remember that MUSICOS uses an echelle spectrograph. Considering this, the efficiency and throughput is comparable. Of course, we lose light with the fibers, but to use a polarimeter at the cassegrain focus and measure in high resolution mode we need them to feed a coudé spectrograph.

F. Vakili: The next step to spectropolarimetric observations is to add spatial resolution. With this in mind, we have equipped our GI 2 T optical interferometer with polarimetric equipment.

T. Eversberg: Exactly. This is already shown by the Bjorkman/Quirrenbach paper and I think that this should be developed further.

R. Ignace: In your test observations of $53 \mathrm{Cam}$, the $\mathrm{V}$ feature appears to be a marginal detection to my eye (i.e., the error bars are large compared to the feature). If you did not know a magnetic field was present, would you claim to have detected one from your data? And to what confidence level?

T. Eversberg: As far as I remember, the result is from a 5-hour exposure and for this a high level of confidence is not reachable. John Rice modelled our observations, but I do not know the result so far. In general, as I mentioned in my talk, we need big telescopes for detecting circular polarisation in an accurate way.

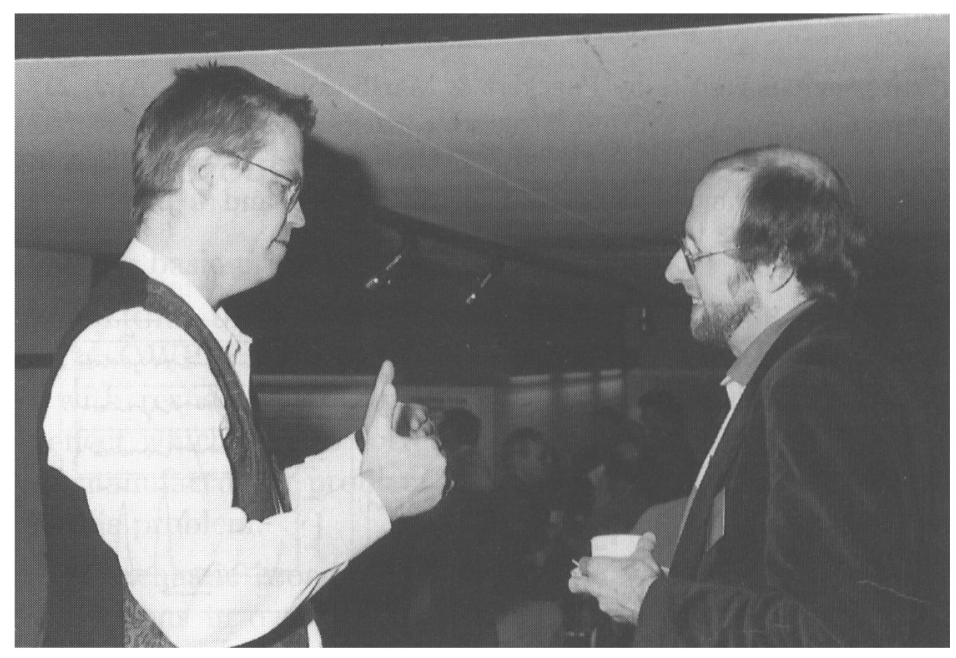

Thomas Eversberg and Gautier Mathys 